\title{
PROfissionalizaçÃ de ATENDENTES de ENFERMAGEM NO ESTAdO de SÅ PAUlo: UM ESTUdo SOBRE A OFERTA E DEMANDA DE FORMAÇÃO
}

Lavínia Santos de Souza Oliveira ${ }^{1}$ Glória Nicoladelli Lampe ${ }^{2}$ Cleide Lavieri Martins ${ }^{3}$ Sueli Yuriko Miyashiro ${ }^{4}$

Oliveira LSS, Lampe GN, Martins CL, Miyashiro SY. Profissionalização de atendentes de enfermagem no Estado de São Paulo: um estudo sobre a oferta e demanda de formação. Rev Latino-am Enfermagem 2002 setembro-outubro; 10(5):637-43.

Este estudo exploratório objetivou contextualizar a problemática de profissionalização dos atendentes de enfermagem e analisar a oferta de cursos e a demanda de formação de nível técnico de enfermagem em São Paulo. As informações foram obtidas a partir de levantamento documental sobre qualificação profissional na área, nos anos 90. Identificou-se um cenário de mudanças conceituais e políticas de profissionalização e a existência de grande contingente de atendentes a serem profissionalizados. Verificou-se uma tendência de aumento na oferta de cursos e na demanda para a formação e as especializações de nível técnico.

DESCRITORES: educação técnica em enfermagem, recursos humanos de enfermagem, profissões em saúde

\section{PROFESSIONALIZATION OF NURSING AIDES AT THE STATE OF SÃO PAULO, BRAZIL: A STUDY ON OFFER/DEMAND REGARDING CAPACITATION}

The purpose of this exploratory study was to verify the problem of nursing aides professionalization and to analyze the offer of courses and demand regarding technical level capacitation at the state of São Paulo, Brazil. Data were obtained through the analysis of documents on professional capacitation in the area in the 1990s. Authors identified a scenario of conceptual and political changes regarding the professionalization and the existence of a great number of aides being professionalized. They also verified a tendency to increase the offer of courses and the demand regarding the capacitation and specialization at the technical level.

\section{PROFESIONALIZACIÓN DE AYUdANTES DE ENFERMERÍA EN EL ESTAdO de SÃo PAULO: UN ESTUdio SOBRE LA OFERTA Y DEMANDA DE FORMACIÓN}

Este estudio, de carácter exploratorio tuvo por objetivo contextualizar la problemática de profesionalización de los ayudantes de enfermería y analizar la oferta de cursos y la demanda de formación de nivel técnico de enfermería en São Paulo. La información fue obtenida a partir de un levantamiento documental sobre la cualificación profesional en el área en los años 90. Se identificó un escenario de cambios conceptuales y políticas de profesionalización y la existencia de un gran contingente de personal a ser profesionalizado. Hay una tendencia de aumento en la oferta de cursos y en la demanda para la formación y las especializaciones de nivel técnico.

DESCRIPTORES: educación técnica en enfermería, personal de enfermería, profesiones de salud

\footnotetext{
${ }^{1}$ Doutoranda em Saúde Pública, Enfermeira da Secretaria Municipal de Saúde da PMSP comissionada na Faculdade de Saúde Pública da Universidade de São Paulo, e-mail: lavinia@usp.br; ${ }^{2}$ Enfermeira com Especialização em Enfermagem de Saúde Pública; ${ }^{3}$ Doutor em Saúde Pública, Professor Doutor da Faculdade de Saúde Pública da Universidade de São Paulo, e-mail: clcide@usp.br; ${ }^{4}$ Enfermeira com Especialização em Enfermagem de Saúde Pública, Técnica do Centro de Formação dos Trabalhadores de Saúde da Secretaria Municipal de Saúde da PMSP
} 


\section{INTRODUÇÃO}

A formação de recursos humanos para a saúde em nosso país consiste em um dos maiores desafios para a organização do setor. Há um déficit crônico de profissionais técnicos qualificados, situação que coloca em risco a assistência à saúde da maior parte da população. Dentre as causas dessas problemática podemse destacar as distorções do mercado de trabalho, que restringe a oferta de empregos para técnicos, utilizando força de trabalho não qualificada e sem formação específica, a crise de financiamento do setor público, as dificuldades de regulamentação do exercício profissional e a carência de propostas educativas que respondam às demandas específicas ${ }^{(1)}$.

Dentre os trabalhadores de saúde é expressiva a representatividade numérica da enfermagem, e são muitas as possibilidades de preparo para os trabalhadores não qualificados que estão em atividade no setor. Para o Conselho Federal de Enfermagem (COFEN), o atendente corresponde à "categoria não formalmente preparada pelo sistema de ensino ${ }^{(2)}$. Recebe alguma forma de treinamento em serviço nas várias instituições de saúde onde executa tarefas de enfermagem, nem sempre devidamente supervisionadas" ${ }^{\prime 3)}$.

Atendentes de enfermagem e assemelhados passaram a integrar legalmente o quadro de enfermagem apenas em 1976, através da Resolução COFEN-18/76. A lei vigente na época, $n^{\circ} 2604 / 55$, que dispunha sobre o exercício profissional da enfermagem, não havia incluído esses trabalhadores. Por assemelhados, entende-se: ajudante de ambulância, atendente rural, auxiliar hospitalar, auxiliar de maternidade, auxiliar operacional de serviços diversos, auxiliar de puericultura, auxiliar de serviços médicos, orientador de saúde, parteira curiosa, samaritana, monitor de saúde, socorrista, voluntária de creche, e outros correspondentes que exercem trabalho remunerado e do mesmo nível de atribuição.

Tentativas de profissionalização dos atendentes de enfermagem não são recentes e resultaram em experiências de diferentes impactos na qualidade da assistência à saúde. Quantitativamente, porém, nunca houve uma solução para esta problemática no Brasil e América Latina*.

A formação profissional de enfermagem está regulamentada no país, desde 1949. Desde essa data, têm sido constantes os esforços governamentais das esferas da educação e saúde e também da iniciativa privada para viabilizar a profissionalização dos trabalhadores de enfermagem ${ }^{(4)}$.

A legislação vigente, $n^{\circ} 7.498 / 86$, em relação ao trabalho dos atendentes, determina que:

"O pessoal que se encontra executando tarefas de enfermagem em virtude de carência de recursos humanos de nível médio nessa área, sem possuir formação escolar específica regulada em lei, será autorizado, pelo Conselho Federal de Enfermagem, a exercer atividades elementares de enfermagem".

Estabeleceu-se, ainda, no parágrafo único deste artigo, que a autorização fosse concedida no prazo de dez anos, expirada em 1996.

Na prática, o sistema de saúde continuou a absorver os atendentes de enfermagem, embora em São Paulo a categoria tenha decrescido em 29\%, entre 1983 e $1996^{(5)}$. A despeito das demandas sociais e imposições legais, observa-se que há uma grande dificuldade em se precisar o quantitativo de trabalhadores de enfermagem a serem profissionalizados no Estado de São Paulo.

Tal dificuldade deve-se em parte à extensa rede de serviços a qual inclui o trabalho de enfermagem.

As iniciativas de qualificação profissional para trabalhadores da área de saúde vêm sendo impulsionadas desde o final da década de 80, quando da implantação do Sistema Único de Saúde - SUS. Nesse cenário, destacase o trabalho das escolas técnicas de saúde (ETS), que foram criadas para exercer papel estratégico na profissionalização de trabalhadores de nível médio. A criação das escolas visou institucionalizar um projeto nacional de formação profissional para trabalhadores empregados nos serviços de saúde, que desempenhavam variadas funções. Esse contingente representava $50 \%$ da força de trabalho em saúde ${ }^{(6)}$.

Ressaltamos que a profissionalização passa pelo acesso ao ensino fundamental. Mesmo sendo a rede de escolas e cursos de enfermagem em São Paulo aparentemente extensa, e as possibilidades de formação, variadas, são poucas as instituições que têm condições de executar a política educacional vigente e responder às novas demandas sociais, como, por exemplo, integrar o ensino fundamental ao técnico, garantir a progressividade curricular, oferecer especializações para o nível técnico e preparar docentes especificamente para a formação do

* Conselho Federal de Enfermagem - Documentos Básicos do COFEN, Resolução COFEN n 18/84, RJ, p. 53 
aluno trabalhador integrado ao contexto do ensino/ trabalho.

Neste momento, entrecruzam-se uma nova orientação educacional para a formação profissional e as necessidades de formação dos recursos humanos pelo Sistema de Saúde. As demandas de profissionalização de trabalhadores da saúde vêm sendo atendidas pelos órgãos competentes que têm procurado diferenciar as políticas de educação profissional, buscando, para sua execução, apoio financeiro de fontes financiadoras internacionais $^{(7)}$.

Uma das estratégias recentes adotadas pelo Ministério da Saúde é o Projeto de Profissionalização dos Trabalhadores de Enfermagem (PROFAE), que prevê profissionalizar 400 mil atendentes e obter impacto na melhoria da qualidade dos serviços prestados à população $^{(8)}$.

As autoras, em seu trabalho, assessoram centros formadores, participando da elaboração de projetos de profissionalização de enfermagem, no âmbito do estado de São Paulo. Faz parte do seu cotidiano analisar a oferta e demanda para cursos de formação profissional. A execução dessa pesquisa decorreu da necessidade de aprofundar a discussão das seguintes questões: Quantos são os atendentes de enfermagem que precisam ser profissionalizados? Quantos cursos de auxiliar de enfermagem existem e quais características têm? Atendem às necessidades e ao perfil dos trabalhadores ainda não qualificados?

São objetivos deste estudo descrever o contexto da profissionalização de atendentes de enfermagem no estado de São Paulo, analisar a dinâmica da oferta de cursos no final da década de 90 e evidenciar as demandas e tendências de formação para a educação profissional de nível técnico, na área de enfermagem.

\section{PROCEDIMENTOS METODOLÓGICOS}

A pesquisa caracterizou-se como um estudo exploratório. A finalidade de um estudo exploratório é esclarecer ou precisar o problema e ainda elaborar hipóteses que possam dar subsídio a estudos posteriores $^{(9)}$. Durante as atividades de consultoria a centros formadores, nos anos de 1999 e 2000, as autoras tiveram a oportunidade de conhecer o universo de trabalho de diferentes interlocutores institucionais.

Para o delineamento inicial da pesquisa, foram contatadas entidades e dirigentes responsáveis por centros formadores na área da saúde e de enfermagem das seguintes instituições: Conselho Regional de Enfermagem de São Paulo - COREN SP, Sindicato dos Trabalhadores Públicos de Saúde do Estado de São Paulo -SINDSAÚDE, Centro de Formação dos Trabalhadores de Saúde da Secretaria Municipal de Saúde de São Paulo - CEFOR/ PMSP, Centro de Desenvolvimento de Recursos Humanos da Secretaria de Estado da Saúde de São Paulo CEDRHU/SESSP, Centro Estadual de Educação Tecnológica Paula Souza - CEETEPS e Sindicato dos Empregados de Estabelecimentos de Saúde. A escolha das instituições participantes do estudo foi orientada por critérios de relevância, tradição, abrangência geográfica e impacto sobre a formação de auxiliares de enfermagem no estado.

Buscou-se identificar, nas instituições, documentação existente sobre a educação profissional de enfermagem. Os escassos bancos de dados disponíveis evidenciaram maneiras diversas de compor e tratar dados nas diferentes instituições, razão pela qual a investigação direcionou-se para um trabalho mais detalhado com informações geradas no ano de 1999 em apenas duas delas:

- Conselho Regional de Enfermagem de São Paulo COREN SP, que registra o acompanhamento da movimentação dos cursos de nível técnico autorizados na área de enfermagem no estado, coletando sistematicamente, em planilhas específicas, informações do Diário Oficial do Estado de São Paulo.

- Secretaria Estadual do Emprego e Relações do TrabalhoSERT, do governo do Estado de São Paulo, que, em parceria com mais de uma dezena de instituições, tem disponibilizado via Internet informações sobre políticas de emprego e renda no estado*. Os dados sobre entidades de educação profissional na área de saúde, utilizados neste estudo, partiram de informações de mais de 200 municípios, relativas ao ano de 1999, encontram-se compiladas em CD-ROM.

A reflexão das autoras baseada na análise desses documentos, na bibliografia pertinente, e nas anotações pessoais durante os trabalhos de assessoria, permitiram discutir a questão deste estudo, focada no período do final da década de 90.

\footnotetext{
* SERT - homepage www.emprego.sp.gov.br
} 


\section{RESULTADOS E DISCUSSÃO}

Situação dos atendentes de enfermagem no Estado de São Paulo

Os atendentes de enfermagem já ocuparam quantitativamente a maior força de trabalho em enfermagem no país e em São Paulo. Segundo dados do COFEN (Conselho Federal de Enfermagem), em 1982, correspondiam a $67 \%$ da força de trabalho em enfermagem no Estado de São Paulo, os auxiliares de enfermagem $21,2 \%$, os técnicos $3,5 \%$ e os enfermeiros $8,3 \%$. Em 1996 , o Conselho Regional de Enfermagem de São Paulo COREN SP e a Associação Brasileira de Enfermagem (ABEn) pesquisaram a força de trabalho em enfermagem no estado e os dados obtidos indicavam que já havia inversão neste quadro. Os auxiliares ocupavam $47 \%$ da força de trabalho e os atendentes $38 \%$, seguidos por $11 \%$ de enfermeiros e $4 \%$ de técnicos de enfermagem ${ }^{(10)}$. Em números absolutos, no entanto, os atendentes continuavam tendo uma grande relevância. A Tabela 1 apresenta a evolução do quantitativo dos profissionais de nível médio de enfermagem, segundo informes do CORENSP, divulgados em boletins, nos anos de 1996 a 1999.

Tabela 1 - Evolução do quantitativo do pessoal de enfermagem em São Paulo, no período de 1996, 1998 e 1999

\begin{tabular}{|c|c|c|c|c|c|c|}
\hline \multirow{2}{*}{ Profissional } & \multicolumn{2}{|c|}{1996} & \multicolumn{2}{|c|}{1998} & \multicolumn{2}{|c|}{1999} \\
\hline & $\mathrm{N}^{\circ}$ & $\%$ & $\mathrm{~N}^{\circ}$ & $\%$ & $\mathrm{~N}^{\circ}$ & $\%$ \\
\hline Enfermeiro & 11.581 & $11 \%$ & 22.060 & $10,1 \%$ & 23.670 & $10 \%$ \\
\hline Técnico de enfermagem & 4.297 & $4 \%$ & 12.365 & $5,9 \%$ & 14.562 & $6 \%$ \\
\hline Auxiliar de enfermagem & 49.772 & $47 \%$ & 116.142 & $53 \%$ & 137.290 & $56,4 \%$ \\
\hline Atendente de enfermagem & 40.339 & $38 \%$ & 67.333 & $31 \%$ & 67.154 & $27,6 \%$ \\
\hline Total & 105.989 & $100 \%$ & 217.900 & $100 \%$ & 242.676 & $100 \%$ \\
\hline
\end{tabular}

Fonte: Compilado de boletins do Conselho Regional de Enfermagem de São Paulo de 1996 a 1999

Não só o aparelho formador, mas também os serviços de saúde buscaram quantificar e qualificar a sua força de trabalho em enfermagem, a partir do ano de 1986. A Secretaria Municipal de Saúde de São Paulo, promoveu, em 1989, um levantamento do seu quadro de enfermagem e constatou a existência de 3.974 atendentes. No ano de 1999, identificaram-se 600 atendentes na rede de serviços públicos municipais*. Essa mudança reflete, em parte, a política adotada de formação profissional desenvolvida na última década. A Secretaria de Estado da Saúde, por meio da Coordenadoria de Recursos Humanos, identificou, em seu quadro de funcionários, 5.118 atendentes de enfermagem, no ano de 1999**. Estima-se que muitos já concluíram cursos de enfermagem nos diversos níveis, mas não tiveram oportunidade de ascensão funcional. Os levantamentos realizados na década de 90 vêm apontando um crescimento vertiginoso dos auxiliares de enfermagem e, embora proporcionalmente inferiores, uma elevação do número de atendentes autorizados ${ }^{(11-12)}$.

Em 1998, o COREN-SP indicava existência de 36.000 atendentes autorizados e 17.000 não autorizados e informava que o número total de cadastrados correspondia a 67.333 atendentes de enfermagem (31\%) e 116.142 auxiliares de enfermagem ou 53\% desta mesma força de trabalho ${ }^{(13)}$. Segundo a Resolução COFEN-185, o atendente autorizado é o portador da cédula que the permite executar atividades elementares na área de Enfermagem ${ }^{(12)}$. Em outubro de 1999, o COREN SP informava*** que, apesar de terem sido cadastrados mais 21.148 auxiliares de enfermagem no período, totalizando 137.290 trabalhadores, o número de atendentes de enfermagem tinha decrescido em apenas 179 trabalhadores. Os quantitativos de enfermeiros e técnicos de enfermagem continuavam numericamente inferiores, mantendo a percentagem de $6 \%$ para estes, e $10 \%$ para os enfermeiros.

Segundo a Associação Brasileira de Enfermagem - Seção São Paulo (1999) ${ }^{\star \star * *}, 80.000$ atendentes de enfermagem estariam na meta de qualificação do Governo Federal pelo PROFAE. O cadastramento espontâneo de atendentes, realizado por esse Projeto, em 2000, identificou 28.532 atendentes a serem profissionalizados.

\footnotetext{
* Prefeitura do Município de São Paulo. Secretaria Municipal de Administração. Relatório Quantitativo, 1999. ASPLAN/Informática, nov. 1999

** Secretaria de Estado da Saúde. CEDRHU. Situação de Atendentes de Enfermagem por DIR, 1999

*** Conselho Regional de Enfermagem de São Paulo. Boletim Informativo. São Paulo, nov. 1999

**** Associação Brasileira de Enfermagem. Informativo Paulista. São Paulo, nov. 1999. (115)
} 
No mesmo período, o COREN-SP informou a existência do cadastro de 16.754 atendentes, mantendo a disparidade entre as informações sobre o registro dessa categoria.

Esse conjunto de dados, ainda que não seja conclusivo, evidencia que se mantêm elevados os números de atendentes, apesar do ingresso de novos auxiliares no mercado de trabalho, o que nos leva a presumir que:

- embora exista uma grande oferta de cursos na rede pública e privada, além dos programas específicos para formação dos trabalhadores, eles não têm produzido impacto suficiente para diminuir o número de trabalhadores não qualificados de enfermagem;

- com a expansão do processo fiscalizatório, o COREN tem identificado atendentes de enfermagem com início da atividade anterior a 1986, pois, segundo a pesquisa sobre Força de Trabalho em Enfermagem, no Estado de São Paulo, $63,7 \%$ dos atendentes ingressaram no mercado de trabalho, antes de $1986^{(10)}$;

- há um movimento dinâmico na vida profissional dos atendentes de enfermagem expresso em aposentadorias, realização de curso de auxiliar de enfermagem, dificuldades para transposição de cargos pelo serviço público e início do exercício de atividades profissionais, como atendente, após 1986. A variação numérica de atendentes em exercício no Estado de São Paulo, conforme a fonte pesquisada, oscilou de 16.754 cadastrados a estimativas de 80.000 .

Os cursos de enfermagem de nível técnico no Estado de São Paulo

Segundo a planilha elaborada pelo COREN SP, no ano de 1999, a Secretaria da Educação autorizou o funcionamento de 223 cursos, sendo 106 de Auxiliar, e 117 de Técnico de Enfermagem (Tabela 2).

Tabela 2 - Cursos de enfermagem, em nível técnico, autorizados em 1999 pela Secretaria de Estado da Educação, segundo regiões do Estado de São Paulo. São Paulo, 2000

\begin{tabular}{lcccccc}
\hline \multirow{2}{*}{ Cursos } & \multicolumn{3}{c}{ Grande São Paulo Interior do Estado } & \multicolumn{2}{c}{ Total } \\
& $\mathrm{N}^{0}$ & $\%$ & $\mathrm{~N}^{\circ}$ & $\%$ & $\mathrm{~N}^{\circ}$ & $\%$ \\
\hline $\begin{array}{l}\text { Auxiliar de } \\
\text { enfermagem }\end{array}$ & 41 & 45,6 & 65 & 48,8 & 106 & 47,5 \\
$\begin{array}{l}\text { Técnico de } \\
\text { enfermagem }\end{array}$ & 49 & 54,4 & 68 & 51,2 & 117 & 52,5 \\
\hline Total & 90 & 100,0 & 133 & 100,0 & 223 & 100,0 \\
\hline
\end{tabular}

Fonte: COREN SP, a partir de publicações do Diário Oficial do Estado de São Paulo em 1999
A proporção entre cursos Auxiliar e Técnico, na capital e interior, está muito próxima. Há um ligeiro predomínio de cursos técnicos (51,2\% no interior e $51,4 \%$ na Grande São Paulo). A capital concentra $17,0 \%$ dos cursos de auxiliar e $16,2 \%$ dos de técnico. Identificaramse alguns projetos especiais de profissionalização: três PROQUALI, de responsabilidade do COREN, e dez classes descentralizadas, de responsabilidade da Secretaria de Estado da Saúde, sendo uma na Administração Penitenciária. Quanto às especializações para o nível técnico, foram identificadas especializações (nomenclatura provisória) autorizadas no ano de 1999, nas áreas de enfermagem do trabalho, pediatria e terapia intensiva.

A totalidade dos cursos é desenvolvida em 147 instituições de ensino, sendo que 60 oferecem tanto auxiliar como técnico. Identificou-se a configuração de "redes" de ensino privado, ou seja, instituições presentes em diferentes regiões, revelando uma tendência que atende à lógica do mercado de ensino. Os dados indicam que a maioria dos cursos é desenvolvida por instituições privadas (Tabela 3), com diferentes entidades envolvidas na formação (Tabela 4): escolas regulares, instituições da administração pública, privada e sindicatos. O investimento privado está se voltando mais para a oferta de cursos técnicos (Tabela 3, 4 e 5).

Tabela 3 - Entidades de Educação Profissional do Estado de São Paulo que ministram cursos na áreas de enfermagem segundo natureza jurídica. São Paulo, 1997 e 1998

\begin{tabular}{lcccc}
\hline Natureza jurídica & \multicolumn{2}{c}{ Auxiliar de Enf. } & \multicolumn{2}{c}{ Técnico de Enf. } \\
& $\mathrm{N}^{\circ}$ & $\%$ & $\mathrm{~N}^{\circ}$ & $\%$ \\
\hline $\begin{array}{l}\text { Privada com fins } \\
\text { lucrativos }\end{array}$ & 35 & 37,2 & 14 & 35,9 \\
$\begin{array}{l}\text { Privada sem fins } \\
\text { lucrativos }\end{array}$ & 43 & 45,8 & 14 & 35,9 \\
$\begin{array}{l}\text { Pública com fins } \\
\text { lucrativos (adm.indireta) }\end{array}$ & 10 & 10,6 & 8 & 20,5 \\
$\begin{array}{l}\text { Pública sem fins } \\
\text { lucrativos (adm.direta) }\end{array}$ & 6 & 6,4 & 3 & 7,7 \\
\hline \begin{tabular}{l} 
Total \\
\hline
\end{tabular} & 94 & 100,0 & 39 & 100,0 \\
\hline
\end{tabular}

Fonte: Cadastro de Entidade de Educação Profissional do Estado de São Paulo. Secretaria de Emprego e Relações de Trabalho, 1999 
Tabela 4 - Entidades de Educação Profissional do Estado de São Paulo que ministram cursos na área de enfermagem, segundo tipo de entidade. São Paulo, 1997 e 1998

\begin{tabular}{|c|c|c|c|c|}
\hline \multirow[t]{2}{*}{ Tipo de Entidade } & \multicolumn{2}{|c|}{ Auxiliar de Enf. } & \multicolumn{2}{|c|}{ Técnico de Enf. } \\
\hline & $\mathrm{N}^{\circ}$ & $\%$ & $N^{\circ}$ & $\%$ \\
\hline \multicolumn{5}{|l|}{ Escolas, centros e } \\
\hline $\begin{array}{l}\text { Institutos de ensino } \\
\text { de nível médio }\end{array}$ & 23 & 24,4 & 14 & 35,9 \\
\hline $\begin{array}{l}\text { Instituições privadas } \\
\text { de ensino profissional livre }\end{array}$ & 21 & 22,3 & 5 & 12,8 \\
\hline $\begin{array}{l}\text { Instituições vinculadas a } \\
\text { empresas ou grupos } \\
\text { empresariais }\end{array}$ & 1 & 1,1 & - & - \\
\hline $\begin{array}{l}\text { Instituições vinculadas à } \\
\text { administração pública }\end{array}$ & 9 & 9,5 & 9 & 23,1 \\
\hline Organizações privadas & 2 & 22 & - & _- \\
\hline $\begin{array}{l}\text { sem fins lucrativos e } \\
\text { não representativas }\end{array}$ & & & & \\
\hline $\begin{array}{l}\text { Instituições vinculadas } \\
\text { a confederações, } \\
\text { federações e sindicatos. }\end{array}$ & 34 & 36,1 & 10 & 25,7 \\
\hline $\begin{array}{l}\text { Instituições de ensino } \\
\text { superior }\end{array}$ & 2 & 2,2 & - & - \\
\hline Instituições comunitárias & 2 & 2,2 & 1 & 2,5 \\
\hline Total & 94 & 100,0 & 39 & 100,0 \\
\hline
\end{tabular}

Fonte: Cadastro de Entidade de Educação Profissional do Estado de São Paulo. Secretaria de Emprego e Relações de Trabalho, 1999

Tabela 5 - Entidades de Educação Profissional do Estado de São Paulo que ministram cursos na áreas de enfermagem segundo origem de recursos. São Paulo, 1997 e 1998

\begin{tabular}{|c|c|c|c|c|}
\hline \multirow[t]{2}{*}{ Origem dos Recursos } & \multicolumn{2}{|c|}{ Auxiliar de Enf. } & \multicolumn{2}{|c|}{ Técnico de Enf. } \\
\hline & $\mathrm{N}^{\circ}$ & $\%$ & $N^{\circ}$ & $\%$ \\
\hline Público & 11 & 11,8 & 7 & 17,9 \\
\hline $\begin{array}{l}\text { Público complementado } \\
\text { com doações e subvenções }\end{array}$ & 2 & 2,1 & 3 & 7,7 \\
\hline Público e privado & 3 & 3,2 & 2 & 5,1 \\
\hline Privado & 29 & 30,8 & 27 & 69,3 \\
\hline $\begin{array}{l}\text { Privado complementado } \\
\text { com subvenções }\end{array}$ & 49 & 52,1 & - & - \\
\hline Total & 94 & 100,0 & 39 & 100,0 \\
\hline
\end{tabular}

Fonte: Cadastro de Entidade de Educação Profissional do Estado de São Paulo. Secretaria de Emprego e Relações de Trabalho, 1999

Quais seriam os fatores determinantes dessa situação? Podemos apontar alguns elementos:

- a grande rede de escolas profissionalizantes não se distribui de maneira uniforme no Estado, refletindo as desigualdades regionais que privilegiam os municípios de maior porte;

- a rede de ensino profissionalizante gratuita não tem oferecido vagas suficientes para os trabalhadores de enfermagem de baixa renda, situação agravada pela dificuldade de acesso ao ensino fundamental, requisito básico de acesso aos cursos profissionalizantes;
- a política de recursos humanos na maioria das empresas está voltada para a diminuição dos custos do serviço, o que as leva a contratar mão-de-obra barata e desqualificada; - as raras iniciativas de qualificação para trabalhadores de enfermagem são isoladas e ignoram o potencial de parcerias entre escolas e serviços; e

- o fato de a preocupação com a qualificação profissional em enfermagem ocorrer em muitas instituições de saúde apenas devido às possíveis sanções legais.

As mudanças no cenário da Educação Profissional refletem a concepção presente na Lei n 9394/ 96 de Diretrizes e Bases da Educação Nacional e no Decreto 2208/97, que entendem "a educação profissional integrada às diferentes formas de educação, ao trabalho, à ciência e à tecnologia" como instrumento que conduz "ao permanente desenvolvimento de aptidões para a vida produtiva"(14) ${ }^{(1)}$ Não se percebe mais a educação profissional como um simples instrumento político assistencialista ou tampouco de mero ajuste às demandas de mercado, mas, sim, como um importante veículo de acesso às conquistas tecnológicas da sociedade como um todo. A educação para o trabalho, atualmente, requer a apropriação de um saber tecnológico e a reelaboração da cultura do trabalho ${ }^{(15)}$.

A resolução CNE/CEB $n^{\circ}$ 04/99 apresenta as seguintes diretrizes curriculares nacionais para a educação profissional de nível técnico:

- desenvolvimento de competências, estando intimamente relacionadas à idéia de laboralidade e "navegabilidade" entre as várias ocupações;

- flexibilidade e interdisciplinaridade curricular, possibilitando a estruturação modularizada dos cursos, com conteúdos de diferentes naturezas, para ampliar a relação entre ensino e trabalho. Assim, os conhecimentos vão se inter-relacionado e se completando; e

- permanente atualização dos cursos, currículos e perfil profissional de conclusão, considerando as demandas locais e regionais, o efetivo preparo para enfrentar desafios e situações novas e que atendam à dinâmica do mundo de trabalho.

A necessidade de adequar o formato dos cursos e sua oferta a essas novas demandas de formação, individuais, institucionais e do mercado de trabalho consiste em um desafio.

A formação do auxiliar de enfermagem está temporariamente inserida no nível técnico da educação profissional, com a possibilidade de progressão para o técnico de enfermagem. 


\section{CONSIDERAÇÕES FINAIS}

A análise primeira destas informações evidencia que a profissionalização dos atendentes foi se dando, desde 1986, por meio da intensificação de cursos supletivos. A demanda para os cursos ocorreu de forma individual e institucional, tanto na esfera pública quanto na privada. As estratégias institucionais adotadas foram a integração ensino - serviço, convênios com instituições formadoras e de assistência e a descentralização dos cursos.

Observou-se que a implementação de experiências pedagógicas inovadoras, como o Projeto Larga Escala, trouxe a questão da formação profissional para o espaço de trabalho, mas não foi capaz de atender a grande demanda quantitativa da profissionalização. Um levantamento realizado para o PROFAE, em maio de $2000^{*}$, identificou em São Paulo 28.532 atendentes sem qualificação profissional, sendo que $28 \%$ não concluem o ensino fundamental.

A análise dos cursos ofertados indica o predomínio de escolas privadas, que atendem a uma clientela espontânea, com condições de custear os estudos. 0 currículo desenvolvido e as oportunidades de estágio são voltados à área hospitalar.

Observa-se um grande contingente de atendentes de enfermagem concentrados nos serviços de saúde pública de municípios de pequeno porte e nos hospitais

\section{REFERÊNCIAS BIBLIOGRÁFICAS}

1. Oliveira LSS. A profissionalização dos trabalhadores de enfermagem nos serviços de saúde: uma experiência de construção do projeto larga escala na cidade de São Paulo. São Paulo. [dissertação]. São Paulo (SP): Faculdade de Saúde Pública/USP; 1996.

2. Almeida MCP, Rocha JSY. O saber de enfermagem e sua dimensão prática. São Paulo (SP): Cortez; 1986.

3. Santos I, Souza AA. Formação de pessoal de nível médio pelas instituições de saúde: projeto larga escala, uma experiência em construção. Saúde em Debate 1989; (mar):61-4

4. Secretaria de Estado da Educação (SP). Coordenadoria de Estudos e Normas Pedagógicas. Estrutura e funcionamento dos cursos supletivos de qualificação profissional na área de enfermagem. São Paulo: SE/CENP; 1979.

5. Fakih FT, Lesting LS, Reichert MCF, Barbosa DA, Barros ALB. Perfil dos Atendentes de Enfermagem Frente a Lei que Regulamenta o Exercício da Profissão de Enfermagem: Relato de Experiência. Rev Paul Enfermagem 1999 set/dez; 12(3):64-9.

6. Sorio R, Lamarca I. Novos desafios das escolas técnicas do SUS. PHYSYS 1998; 2:125-46. públicos de psiquiatria. Por outro lado, existem, ainda, demandas de formação, partindo de instituições privadas nas quais o trabalho de enfermagem não está sistematizado, como creches, escolas, clínicas geriátricas e serviços de cuidados domiciliares ou home care. Novos estudos são necessários para delimitar a abrangência e caracterizar esses novos campos de atuação profissional.

Em relação à proporção dos cursos autorizados, existe uma certa paridade entre os cursos de auxiliar de enfermagem e de técnicos, indicando uma forte tendência de aumento do segundo.

A busca por especializações em nível técnico já faz parte do itinerário profissional dos alunos, principalmente para a área hospitalar. A enfermagem do trabalho é um campo tradicional de atuação para técnicos, o qual se mantém como opção profissional.

Este estudo revela parcialmente a dinâmica das importantes mudanças pelas quais o mercado de trabalho em saúde e o sistema de educação profissional vêm passando e aponta a necessidade de novos estudos. Parecem urgentes as alternativas de caminhos metodológicos e políticos que aumentem a resolução dos processos de formação, qualifiquem enfermeiros para a formação do aluno trabalhador e atendam ao grande contingente de trabalhadores não qualificados que ainda não conquistaram o direito da cidadania profissional.

7. Leite MCG. Profissionalização dos serviços de enfermagem. Vox Poli 1999; 7:9-12.

8. Ministério da Saúde (BR). Secretaria de Gestão de Investimentos em Saúde. Projeto de profissionalização de trabalhadores de enfermagem - PROFAE. Brasília (DF): Ministério da Saúde; 1999.

9. Gil AC. Métodos e técnicas de pesquisa social. São Paulo (SP): Atlas; 1994

10. Conselho Regional de Enfermagem de São Paulo, Associação Brasileira de Enfermagem - São Paulo. A força de trabalho em enfermagem no Estado de São Paulo. São Paulo (SP); 1996.

11. Dantas RAS, Aguilar OM. O ensino médio e o exercício profissional no contexto da enfermagem brasileira. Rev Latinoam Enfermagem 1999 abril; 7(2):25-32.

12. Conselho Regional de Enfermagem de São Paulo. Principais legislações para o exercício da enfermagem. São Paulo (SP); 1997.

13. Conselho Regional de Enfermagem de São Paulo. Projeto especial de qualificação profissional de auxiliares de enfermagem - PROQUALI. São Paulo (SP); 1998.

14. Souza PND, Silva EB. Como entender e aplicar a nova LDB. São Paulo (SP): Pioneira; 1999.

15. Demo P. Educar pela pesquisa. Campinas (SP): Autores Associados; 1996.

\footnotetext{
* Ministério da Saúde. Secretaria de Gestão e Investimentos em Saúde. Projeto de Profissionalização dos Trabalhadores da Área de Enfermagem. Brasília, 2000
} 